\title{
Communication complexity in high-speed distributed computer network in an agent based architecture for grids service
}

\author{
Serrano, Juan Francisco ${ }^{1 *}$ and Surós Rina ${ }^{2}$ \\ School of Engineering and Applied Sciences, University of East, Monagas, Venezuela \\ School of Computer, Central University of Venezuela, Caracas, Venezuela ${ }^{2}$
}

Received: 15-December-2017; Revised: 17-February-2018; Accepted: 22-February-2018

(C)2018 ACCENTS

\begin{abstract}
Grid is a technology that implements the process of sharing resources in a flexible, secure and coordinated manner. Task management in computational grids involves planning, implementation and monitoring. The main contribution of this work consists in the development of a model with an agent-based architecture for managing computer resources each with defined operations so that the user can perform tasks efficiently and effectively and thus improve substantially the management by a gLite Grid middleware. The solution proposed provides a platform based on a collection of agents in a virtual organization. The model considers the heterogeneity of resources so that it is completely independent of any physical network architecture. This paper focus on the model, simulation and evaluation of an agent-based management of computational resources in grid environment architecture. Experimental results showed significantly the effectiveness of algorithms and planning policies to achieve load balancing, fault monitoring, and service quality. The computational complexity of the proposed model is studied and the experimental results are analyzed with respect to the use of the computing resources.
\end{abstract}

\section{Keywords}

Grids, Agent architecture, Load balancing, Fault monitoring, Computational complexity.

\section{Introduction}

The grid computing infrastructure was developed as a high performance tool for scientific research and cooperation $[1,2]$. It is the dominant technology for large scale resource sharing and integration of distributed systems [3]. It provides an efficient technological platform for network services and applications [4-6].

A computational grid is an infrastructure that enables the interconnection of important distributed computing resources to solve large-scale problems in science, engineering, and trade [7, 8]. Problems due to their high demand for resources cannot be solved with a uniprocessor [9] system. Through administrative domains structuring the grid systems, it is possible to integrate applications, manage resources, coordinate the use of heterogeneous distributed services and guarantee access to dynamic virtual organizations, all in $[10,11]$.

\footnotetext{
*Author for correspondence
}

A virtual organization (VO) is a set of individuals and / or institutions that have direct access to hardware resources, software, data, and tools for collaboration in order to solve problems or for any other purpose. The VO is a concept that facilitates the operation of the grid and is employed to associate users and handling requests and resources. One difficulty of grid computing is the scheduling of geographically distributed resources. Moreover, institutions have different policies for the use of their resources, and groups of people have highly nonuniform performance; they are heterogeneous and have varying loads and availability.

Grid computing goes far beyond the traditional parallel and distributed systems [12]. It involves multiple resources located in different administrative domains and management policies for specific resources in each domain [13]. The gLite grid middleware comes from a series of grid projects: DataGrid [1] DataTag [4], Globus [14-17], and EGEE [18]. It is currently installed at sites participating in the WLCG / EGEE project [18]. GLite infrastructure is a service oriented architecture (SOA) that facilitates interoperability between grid 
services and meets the standard in accordance with the model of open grid service architecture (OGSA) $[17,18]$. Its architecture consists of a set of services and provides the user with an important alignment of services to run computing jobs, access and data movements, and in addition, it provides information on the network. All of these are safety standards for the grid security infrastructure (GSI) [16].
Figure 1, shows the overall architecture of the GLite workload manager (GWM) and its interaction with external entities. The workload manager (WM) and the logging and bookkeeping service to track the events generated by different components for each job that passes through them.

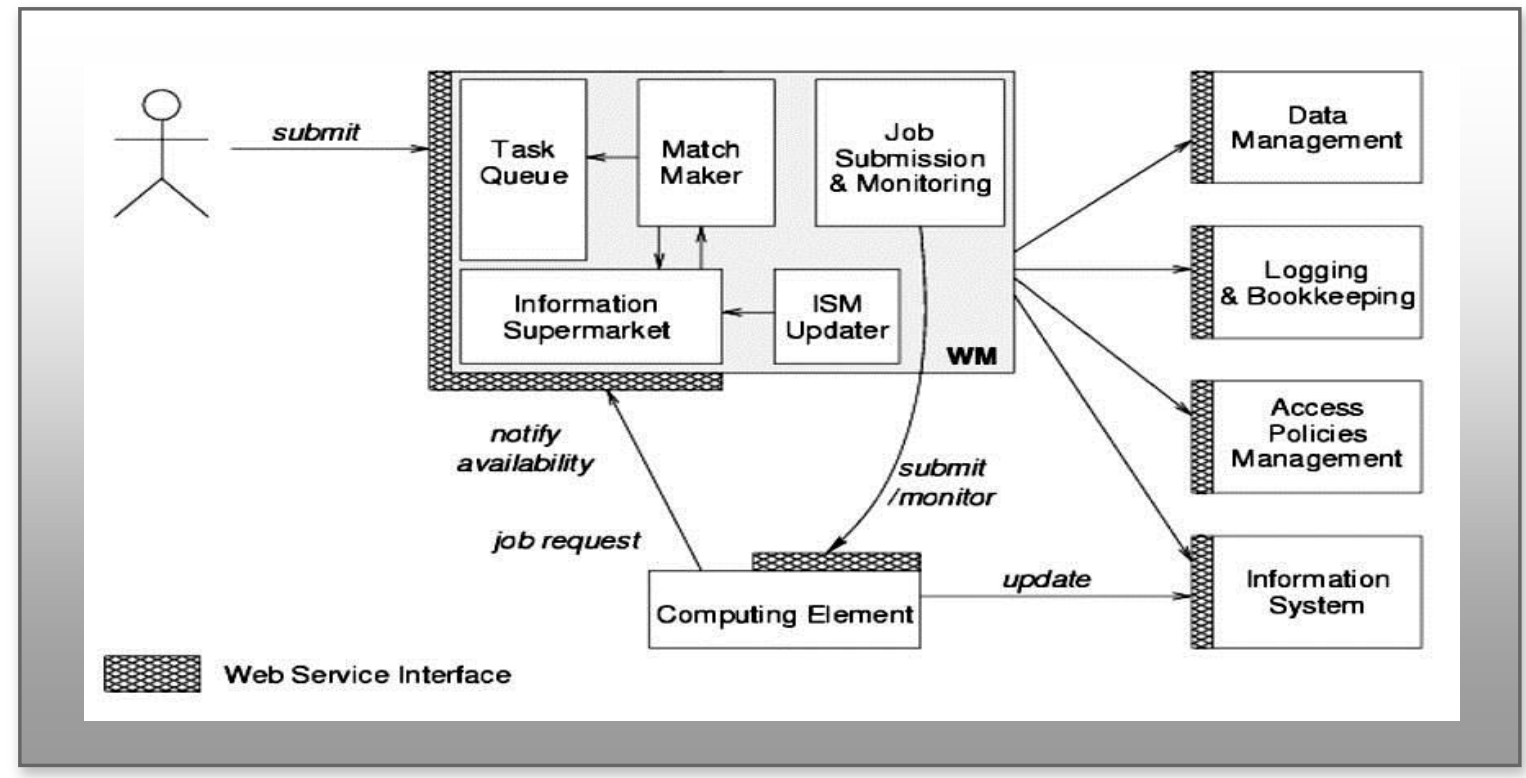

Figure 1 gLite workload manager, internal architecture

The workload manager system (WMS) comprises a set of grid middleware components responsible for distribution and management of tasks by the resources of the grid. This ensures that applications are executed in a convenient, efficient and effective manner $[7,8]$. The specific prototype-computing task required is generally referred to as "job" [16, 17].

Efficiently manage a Grid infrastructure under gLite Grid middleware requires a significant amount of effort in planning to meet the demand for implementation of the multiple job that will share the available resources. A computational grid is an infrastructure that enables the interconnection of computational resources to be shared by multiple users who demand dynamically these resources according to their needs. It considers resources organized by layers. Generally speaking, the layer of highest level requires comprehensive planning tools. Planners do not have direct access or control of resources where the application will run, they select and book (if available) those resources. In the lower-level layer is required to have local resource planners. In addition, the access to resources is shared by several jobs; the heterogeneity of the hardware that makes up the grid is a major problem. A feature of the applications running on the grid is that may require multiple resources for its execution so that its use should be coordinated by multiple administrative domains. The information on the current status of each resource is managed by the local manager at the lowest level, if such information is available at every moment to guarantee the synchronicity between the information published and the real, this is not currently possible. It may happen that a resource that is assigned because it is available in an instant, then it this busy in an instant immediately after the consultation. The dynamic management should assign to each type of application resources that its demand, which makes the selection of critical resources. By the difficulties described in the management resources and job in the existing grid middleware gLite, it needs to be improved in terms of efficiency and flexibility with the implementation of new technologies that will enable the construction of smart grid environments. 
This paper focus on the model, simulation and evaluation of an agent-based management of computational resources in a grid environment, architecture, with the aim of contributing to solve some of the limitations described above. We show the advantages of our proposal for an agent-based resource management compared to the existing gLite grid middleware architecture.

In the system of agents proposed each agent is responsible for a search operation resource monitoring or planning jobs [19]. The communication is managed by the set of agents that resides on the nodes located in each $\mathrm{VO}$ through which requests for resources and by the WMS, so that the deployment of services starts at the point of customer access through the interface provided by the user agent interface. The services of search, discovery, monitoring and resource allocation, is achieved through the interaction of the agents and the tools/services of the WMS. The methodology implemented for the management of resources allows organizing the agents hierarchically. The hierarchy of agents can represent an open and dynamic system where new players can join the hierarchy or get out of it. The hierarchical model solves the problems of scalability. The hierarchy is a logical structure and each one of the agents can communicate with others whenever know their identities. Each agent uses a planning policy to achieve load balancing across multiple local hosts. In the top-level layer using the information provided by the monitoring and discovery mechanism of resources the agents cooperate to balance the load of work.

The assessment of the proposed system of agents is done by simulation techniques. With the simulation model of the gLite middleware, reference values will be generated. Under the same conditions, the multiagent system model is constructed also by simulation techniques. For the evaluation of the models we run the simulators, for both models, using exactly the same set of values of selected parameters with the same data distributions. With the data obtained by both simulations, we do a comparative analysis. The experimental results show the effectiveness of the system of agents proposed with the various policies of planning. So, on achieving load balancing and fault monitoring in grid in an efficient manner, as opposed to the management carried out by the gLite middleware. The analysis of the complexity of the proposed multiagent system shows that the cost of communication complexity is $\leq \mathrm{O}\left(\mathrm{n}^{2}\right)$, if it is assumed that each processor has an unlimited computational power. The communication model is asynchronous and reliable. Confirm so this new paradigm can be used for getting a load of work effectively during the management of resources. This paper has been organized in the following way: section 2 begins by laying out the developing of the multiagent based architecture model for managing computer resources in a Grid. This includes planning, implementation and monitoring for improving the gLite middleware services for tasks involving running in a grid. Also, we assessed the CommonKADS methodology for agents modeling and the computational time complexity of the model. In the section 3 we describe the simulation strategy, the different configurations of a multiagent system, the parameters monitored and the experimental validation. A detailed study of the impact of incorporating strategies in allocation policies jobs in the overall performance of the grid is given, as well as contributions of the research work that is going to be proposed and discussed in Section 4. In the final section, conclusion is given.

\section{Agents and multiagents systems}

Agents are modeled with a MAS-CommonKADS methodology [20, 21]. This is an extension of the CommonKADS multiagent methodology that adds relevant integrating object-oriented techniques and some aspects of protocol design. It provides an object-oriented multi-agent system for structuring notation. It uses the use case to capture requirements and protocol engineering techniques to specify interactions between agents. In total, it uses seven models for the development of multi-agent systems. In the conceptualization phase an introduction to the system with use case based techniques on the users are made. In this manner, the deployment and integration of system components and the software development process are made easier. Moreover, it achieves adaptation of multiagent system for complex, dynamic and geographically distant means. It guarantees safety management jobs.

\subsection{Structure of the platform for agents}

2.1.1The proposed agent-based architecture

The emergence of SOA and the growing interest in supporting a wide range of commercial applications have driven significant advances in the capabilities of resource management [22]. For this purpose, the job is defined as an object running on the network in order to solve a given problem. The properties of the object are ID, name and type, etc. Locate resources to use, mobilize the file to be executed, and coordinate 
the input and output of data or files. The methods of operation include the definition, presentation, planning, search, implementation, and the monitoring of a job [23, 24]. Analysis of the computational complexity of the proposed model allows for a quantitative estimate of the amount of resources required to perform a calculation and the cost of the required communications. The proposed solution is to provide a platform based on a collection of agents in a VO $[15,25]$. The agents are activated when a user authenticates. Their life cycle ends when the application execution completes or aborts and the information is transmitted to the user. In Figure 2, a diagram of the proposed architecture for an agent based approach is shown.

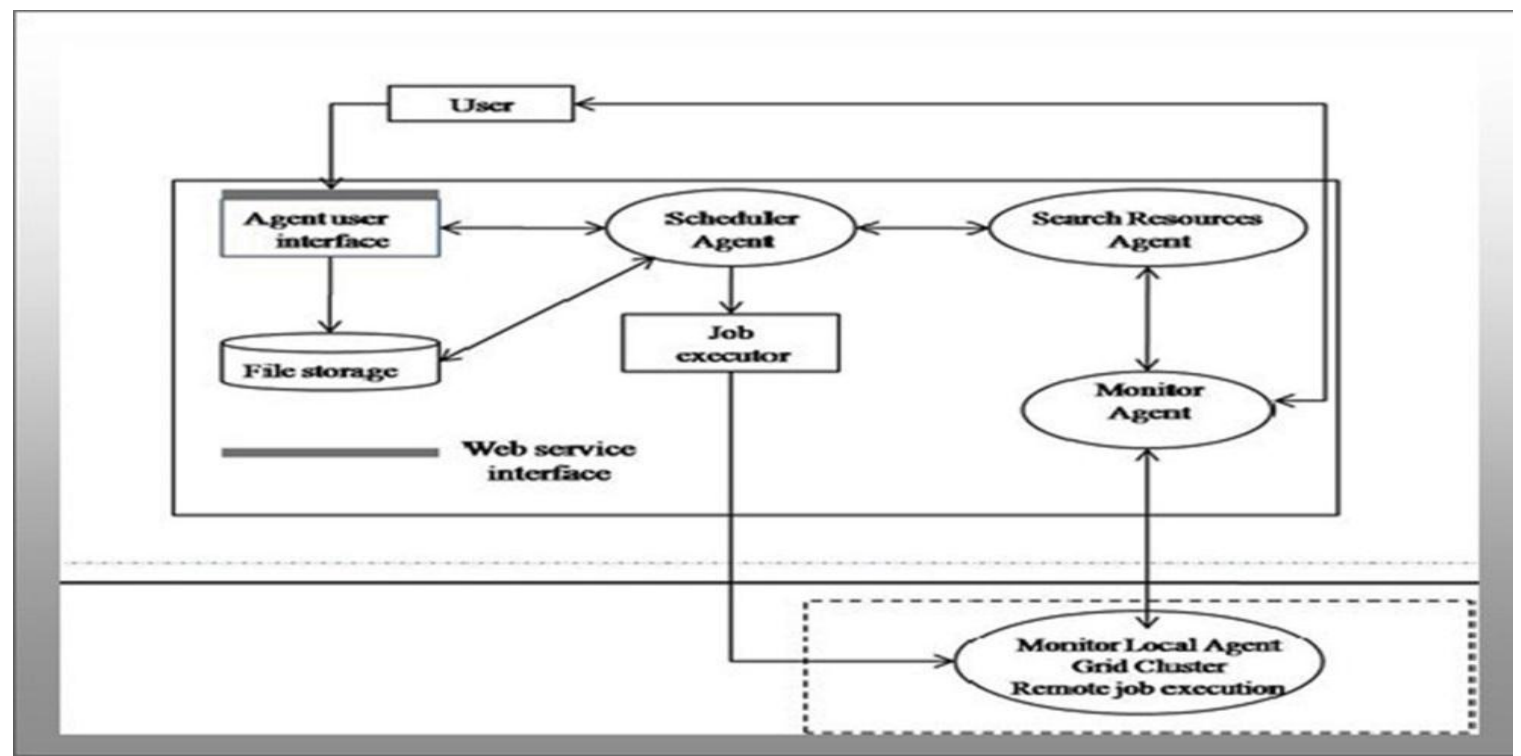

Figure 2 Flowchart of the proposed architecture for an agent based approach

\subsubsection{Communicating agents}

The communication between the agents of the system is achieved by the use of an agent communication language (ACL) recommended by the Foundation for intelligent physical agents (FIPA) [26]. The sequence diagram shown in Figure 3, depicts the message delivery mechanism between the system components when an invocation of the services of the grid takes place [27-30].

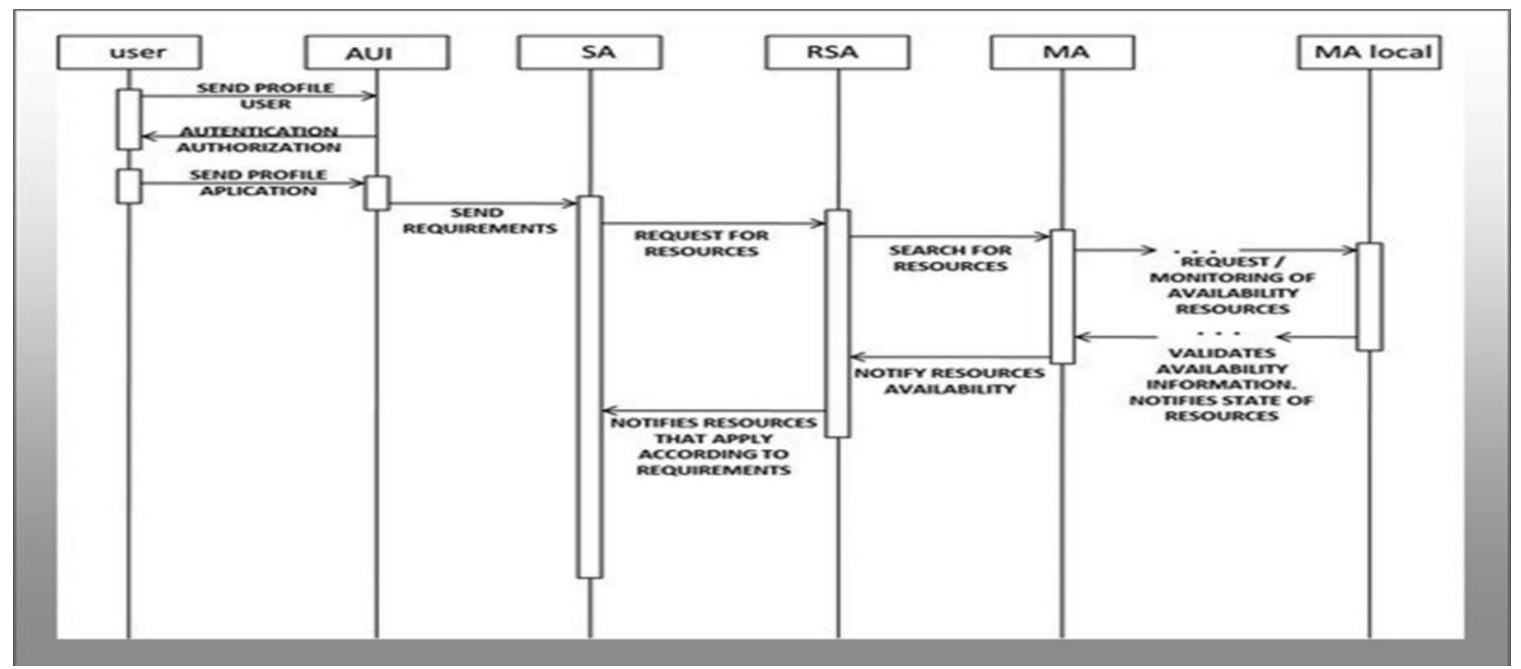

Figure 3 Diagram sequence communications between system agents 
Francisco et al.

2.1.3General architecture of the agent system

In Figure 4, we show the overall system architecture of agents. In Figure 5, we present the intervention of the agent platform with middleware gLite. The gLite middleware layer shows the overall architecture of the WM and its interaction with external entities [31]. The logging and bookkeeping service handles events generated by different components for each running task. The status of the job is the set of events that are generated for processing. The system of agents locates the nodes of each VO resource and manages communication.

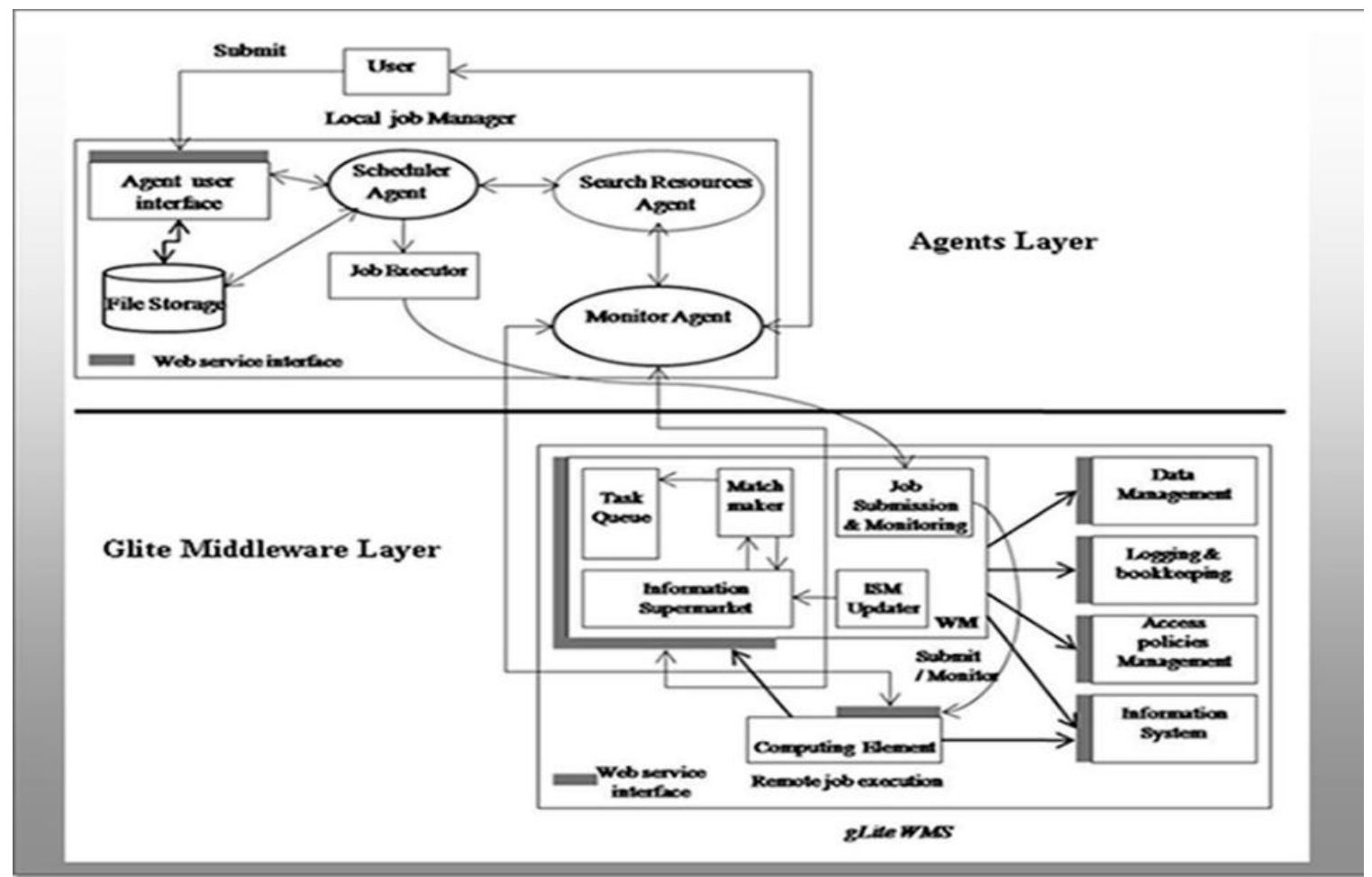

Figure 4 Overall system architecture under control gLite middleware

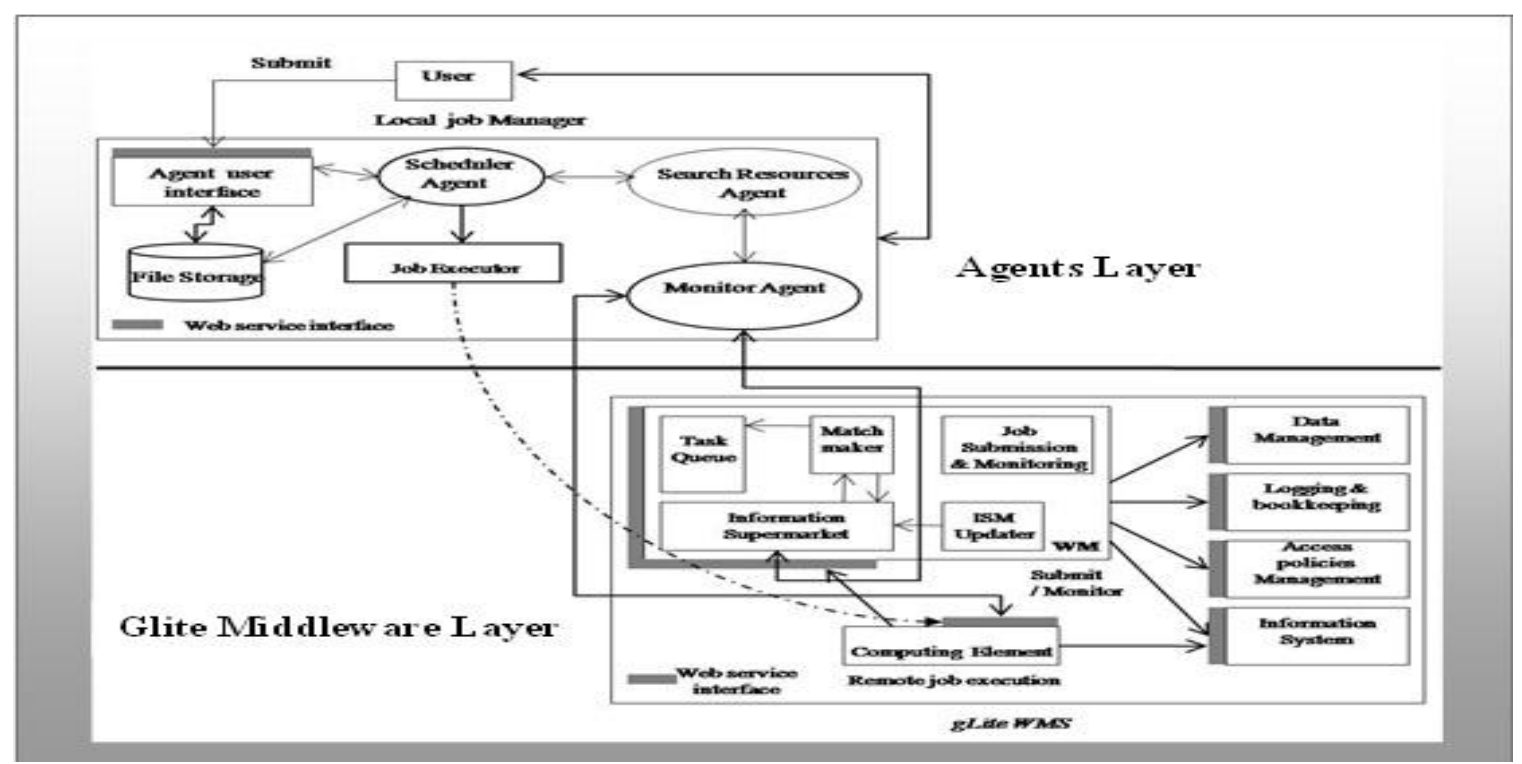

Figure 5 Overall architecture proposed multi agent system that integrates 
The key aspects in the efficient development of the architectural proposal are: resource monitoring, hierarchy of agents, and load balancing. We consider agents with the following functionalities: monitoring, search of resources and planning tasks corresponding to some elements of the gLite WMS [18] services [32]. The client initiates the deployment of services using the interface provided by the agent user interface (AUI). Search services, discovery, monitoring and resource allocation are achieved by the interaction of agents and tools that are part of the WMS. It defines each of these agents.

The agents that constitute this platform are defined as AUI, scheduler agent (SA), searcher resource agent (RSA), and monitor agent (MA).

Agent user interface (AUI): using a web interface for communication with the user, the AUI collects basic information about the type of job resource requests or special needs for the resources. It provides the user with a private certificate for authentication. It sets security mechanisms through the gLite middleware and the protocols GSI. User information is passed to the file storage (FS) that manages the queue and is responsible for maintaining active pending requests. Once the job is processed, the user receives an ID for subsequent actions such as: request status and even request of your story (sequence of states). The development of this interface is based on the specification OGSA, so that the exchange of messages uses the HTTPS protocol under SOAP.

The user interface allows for the description of the work: nature, type, and special requirements that they may have to run on the resources of the grid. All information to be supplied by the user is described using a specific language: job description idiom (JDL). It is a high-level language based on classified advertisement (ClassAd).

Scheduler agent (SA): The SA is responsible for planning the execution of a job on adequate resources. It communicates with the resource searcher agent (RSA) for a list of available resources. It locates the best resource according to the requirements and availability of the machines.

The SA includes its own resource selection policies. The user is part of a community of VO. From this set of machines, a subset of available resources is selected. The MA is in charge of updating the information service discovery middleware gLite. The
SA selects the resources according to the requirements of the job. Both SA as RSA interaction with gLite WMS through an established communication interface. The gLite WMS is responsible for implementing the functionalities of the agents. The job management policies are adopted by the SA and implemented by the WM.

Searcher resource agent (RSA): Given a list of resources, RSA makes the coupling (matchmaking) between the jobs and the resources. Then the RSA functionality moves to the gLite WM. The user can select the resource or allow it to be carried out in a transparent manner according to established criteria. In [18] the development of the gLite architecture is shown in detail.

Monitor agent (MA): The MA is in charge of monitoring the status of the resources to provide online information on availability and, at the same time, it controls tasks performed by every job. The MA multiagent system interacts through a communication interface, managing to keep current information services discovery and updating the gLite middleware.

2.1.4Communication system complexity

The robustness and scalability of multi-agent system depend heavily on computational properties of their algorithms [5]. Sequential time communication begins with the user request and ends with the allocation of resources. Without loss of generality, for the analysis of computational time complexity, it is assumed that all processes are similar and the operations are performed at the same speed, which may not be the case for clusters. Then, the communication complexity in a distributed network can be defined as the number of bits communicated over all links of the network to solve the problem in the worst possible scenario. Also, it assumes that each processor has unlimited computational power and that calculations are instantaneous.

Consider the architecture shown in Figure 2 for the job management. The cost of a message communication can be calculated as follows:

$C_{\text {com }}=\alpha \times\left(t_{p}\right) \times\left(T_{\text {trasm }}\right)+\beta$

Where $\alpha$ : number of words in the message; tp: word size; $\alpha$ (tp): message; Ttrasm: transmission time to send a data word, $\alpha$ (tp) (Ttrasm): transmission time of a data word, and $\beta$ : message latency in the network. We include the case where the source and destination are not connected directly and the message must go through intermediate nodes. 
$\mathrm{C}_{\text {com }}$ is essentially the time needed to package the message in the source and unpack the message at destination, including the number of intermediate nodes in the path. The term latency is used to describe the total delay in communication and it is assumed to have a constant value. The final communication time will be the summation of all the times of sequential messages of all steps involved in the process.

Assuming that the communication time of all the processes has the same pattern, we consider a single process for analysis. A heterogeneous network composed of different administrative domains is considered. The number of resources is limited. The characteristics of these resources can satisfy the requirements of a particular user. Then, we analyze the complexity of the proposed communication in the worst possible model. We will assume that the cost of execution in the local environment has a high computational complexity $\mathrm{O}\left(\mathrm{n}^{3}\right)$.

2.1.4.1Request for resource allocation

The allocation of resources involves a number of steps: a) initially, the client machine requests the resource; b) a thread is generated from the VO to notify the resource, which then will be assigned. The final computational cost is the sum of all costs of communicating sequential messages involved in the process. The transmission phase involves sending the application (COD Code) and sending the data (data packet length L). The message conveyed considered is structured as follows:

$\alpha\left(t_{p}\right) \times\left(T_{\text {trasm }}\right)+(\beta) \times L+\alpha \times\left(t_{p}\right) \times\left(T_{\text {trasm }}\right)+(\beta)$ $C O D$

$C O D<<L$

Sending the application and data to the assigned resource generates an additional jump. This contrasts with the intermediate nodes in the route. Then we add $M+1$ hops in the parameter $\beta$, where $M$ is the number of intermediate nodes.

2.1.4.2Execution and monitoring

The runtimes and application monitoring overlap without adding computational cost. We assume that each t' units of time in a message between the MA and the user is sent. This message is the size of the minimum word i.e. $\alpha=1$. The cost of communication monitoring messages is obtained by:

$C_{\text {com }}=\left(t_{p}\right) \times\left(T_{\text {trasm }}\right)+\beta$

If every $t^{\prime}$ units of time a message is sent and delivered in total $D$ messages, then the equation $\left(t^{\prime}\right)$ $(D+1)$ represents the number of messages sent between the MA monitoring and AUI during the application execution. Thus the communication cost of monitoring all messages will be:

$C_{\text {com }}=t^{\prime}(D+1)\left(t_{p}\right)\left(T_{\text {trasm }}\right)+\beta$

We assume that communications are reliable. The rate of transmission of messages monitoring is $t /$ min. Monitoring messages include $M$ jumps depending on the return path according to the communication protocol. Then, the total communication time is:

$$
\begin{gathered}
C_{\text {com }}=\left(t^{\prime}\right) \times(D+1) \times\left(t_{p}\right) \times\left(T_{\text {trasm }}^{\prime}\right)+\left(\beta^{\prime}\right) \times\left(t_{p}\right) \\
\left(T_{\text {trasm }}\right)+\beta
\end{gathered}
$$

Where $\left(\beta^{\prime}\right)$ and (T'trasm) refer to the transmission time latency between different VO respectively.

2.1.4.3Transmission of results

Let $L^{\prime}$ be: length result data. Then the communication time of the message will be:

$C_{\text {com }}=\alpha\left(t_{p}\right) \times\left(T_{\text {trasm }}\right)+\left(\beta^{\prime}\right) \times L^{\prime}$

If we make $\mathrm{K}=\alpha$ (tp). After some final expression factorizations, in total, communication time will be defined by the following expression:

$C_{\text {com }}=5 \times(K) \times\left(T_{\text {trasm }}\right)+(\beta)+3+L+C O D+L^{\prime}$ $\mathrm{x}(K) \times\left(T_{\text {trasm }}^{\prime}\right)+\beta^{\prime}$

Both $\mathrm{L}$ and $\mathrm{L}^{\prime}$ represent matrix data with computational complexity $\mathrm{O}\left(\mathrm{n}^{2}\right)$ in the worst case, indicating that the communication complexity of the system is $\leq \mathrm{O}\left(\mathrm{n}^{3}\right)$.

\subsubsection{Fault control}

The resource allocation policies should maximize performance, be resistant to failures, and must ensure the recovery of compute nodes [33]. The service fault control is implemented when the monitoring service reports a disruption during execution [34]. At that time the resource allocation process is restarted or execution resumes from the last checkpoint recorded by the service control fault once the repair operation is completed.

2.1.6Load balancing

The service local load balancing determines whether the local SA should assign the job or external resources should apply. The load balancing algorithms can be simple, such as the policy of first come first served (FCFS) or based on complex algorithms [10, 35]. The proposed load-balancing model considers the heterogeneity of resources regardless of the physical network architecture [35]. The resource allocation algorithms reduce the total time to release the job and overall wait time in the queue; additionally, it maximizes the rate of resource utilization and the load balancing between resource 
providers. The model is based on the logical hierarchy of agents [21], which partially solves the scalability, thus adjusting to the different levels of the complexity of the VOs.

\section{Execution and analysis of the simulation model}

3.1Configuration of simulation

For the simulations, SimEvents, a Blockset in Matlab R2013b version were used. We applied the simulation model event to activate the AUI and to initiate activities in the multi-agent system. Various configurations of a multi-agent system were considered; also we varied parameters such as: time between arrival of jobs, length of service of the EC worker node of different virtual organizations, capacity of queues, number of resources, and the inherent parameters of the probability distributions. Grid scheduling is an NP-complete problem [36]. Therefore, we have to resort to various heuristic policies and strategies to address the problem of achieving efficient workload for adequate resource management.

A set of experiments was designed to test the performance of a resource management mechanism when using an agent-based model (SBA). This had two phases: Phase 1 or the simulation resource management of middleware gLite. These data serve as a reference. Phase 2 or simulation resource management of the SBA in the grid in interaction with the gLite middleware system proposed model. These simulations were made according to the general architecture of the system shown in Figure 5.

\subsection{Experimental validation}

\section{Phase 1}

We considered the following parameter values for the simulation resource management of middleware gLite: Message Size: random; Queue Size in two cases: 1) finite queue, 2) infinite queue; service times: uniform and exponential distributions; and inter arrivals time of the job: uniform and exponential distributions. The length of the information message that was generated was random with a discrete uniform distribution with a minimum length of 6 , and a maximum length of 10 bytes. The service times with uniform distribution used the parameters $\mathrm{min}=$ 0.001 and $\max =0.02$. For the generation of time between arrival of jobs, we use exponential distribution with parameter lamda $=200$ and average $l a m d a^{-1}=0.005$

In this scheme, it is assumed that the update time of the information about the availability and occupation of the resources is established by the gLite middleware. Two types of capacity queues for both organizations were considered. For the finite one when queues are saturated applications that have no place in the queue is not processed and processing is aborted. The same behavior was observed with increasing frequency of respect inter-arrival time. We will consider two VOs of 4 and 8 computational resources respectively to achieve a simple visualization of the results. Figures 6 and 7 contain the MATLAB / SIMULINK model that represents the architecture of the gLite middleware is shown.

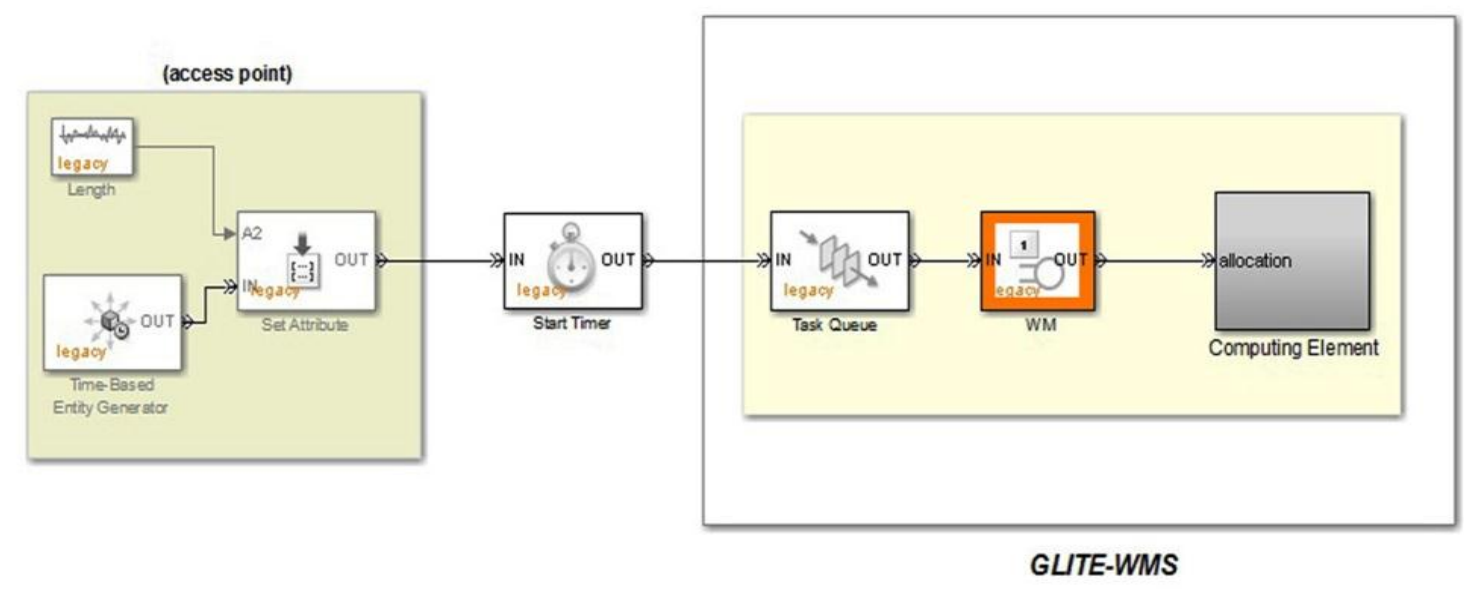

Figure 6 MATLAB/SIMULINK model of the architecture of the gLite middleware 


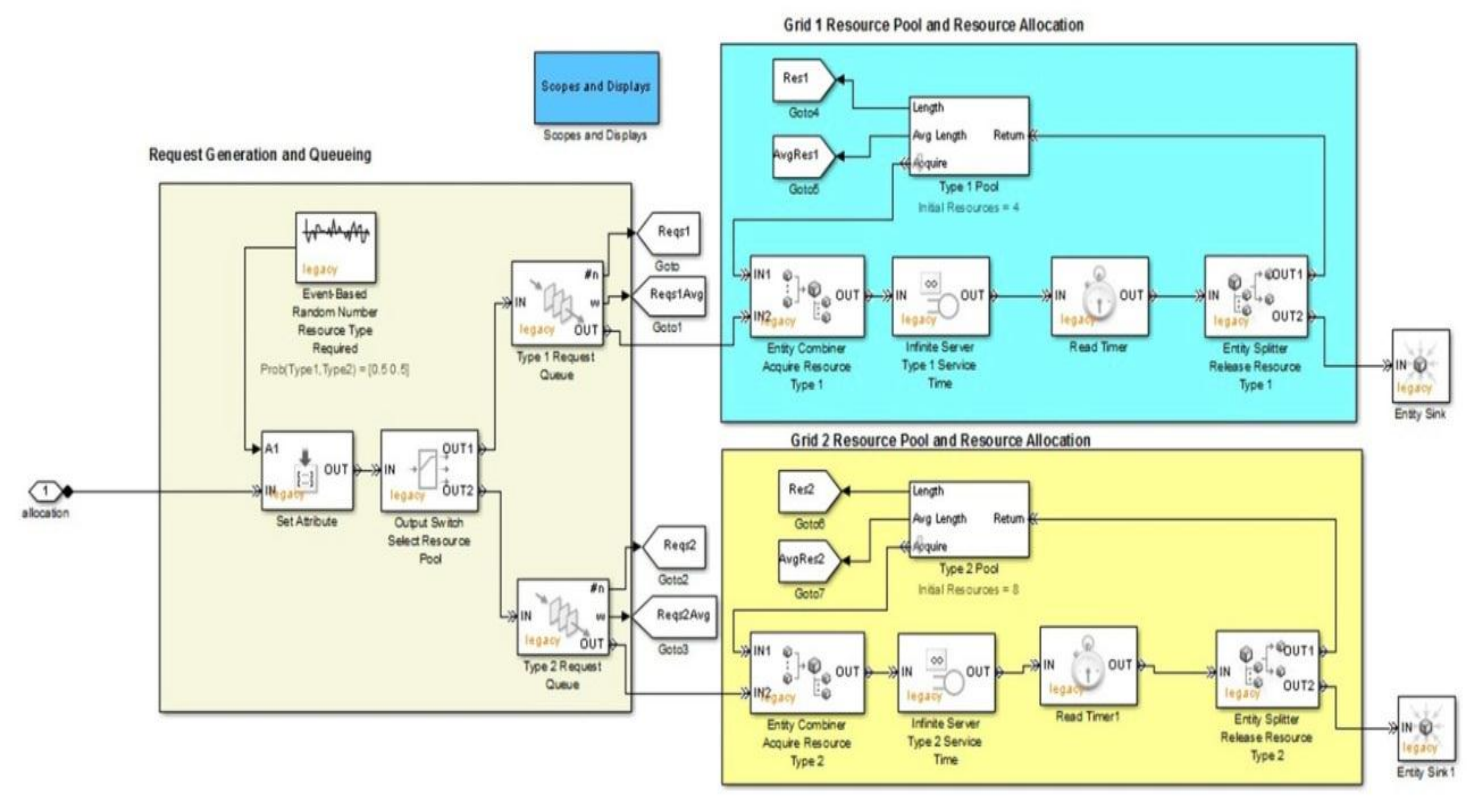

Figure 7 WMS Scheme of the gLite middleware to manage resources in virtual organizations

Figure 7 shows the model of the resource allocation process simulated by MATLAB/Simulink. It describes the policy used: first equipment available, first assigned equipment. Depending on the availability of VO resources known as WMS gLite.

Figure $8 a$ shows the availability of resources for the first VO consisting of a pool of four computational resources. The graph depicts the availability of the resource vs. observation time. In the first 8 time units, we observed the availability of the resource. In the time interval $[8,10]$, the resources were all busy. As the queue size is infinite no resource requests were rejected. When the waiting time of a request by a user is extremely long, the request is aborted. Figure $8 b$ shows the lengths of the queues. In the time interval $[9,10]$ queues were full generating long waiting times because gLite does not handle the information on the availability of online resources in the different VO.

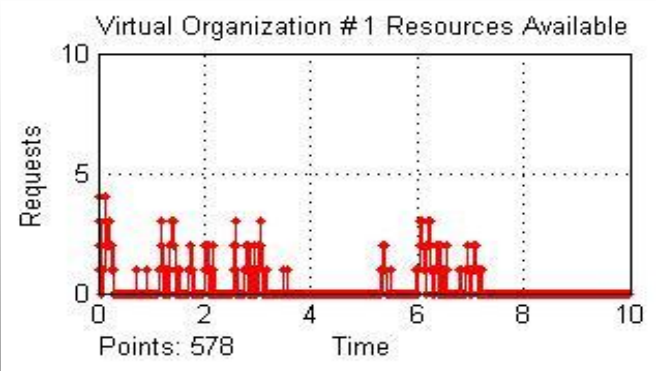

(a)

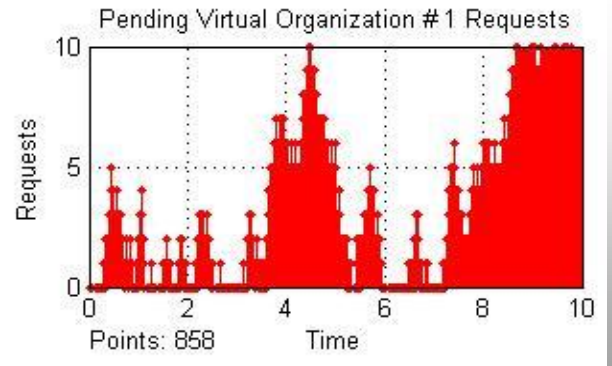

(b)

Figures 8 Description of jobs queued and availability of resources in the VO1

Figures $9 a$ and $9 b$ show the results for the second VO with a pool of 8 computational resources. In time intervals $[1,4]$ and $[5,8]$ high congestion occurred in the queue. This is shown by the limited availability of resources. In the interval [8, 10], there was congestion in the queues. 


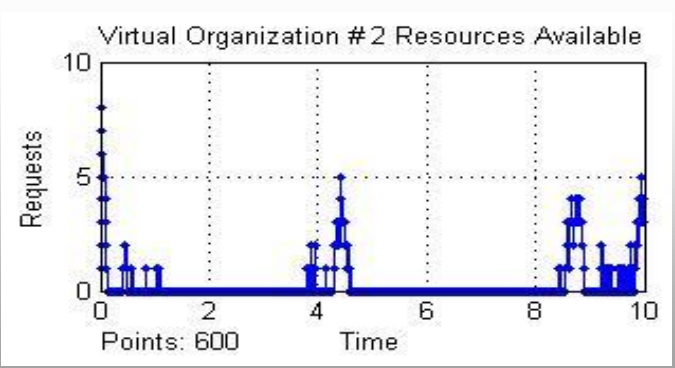

(a)

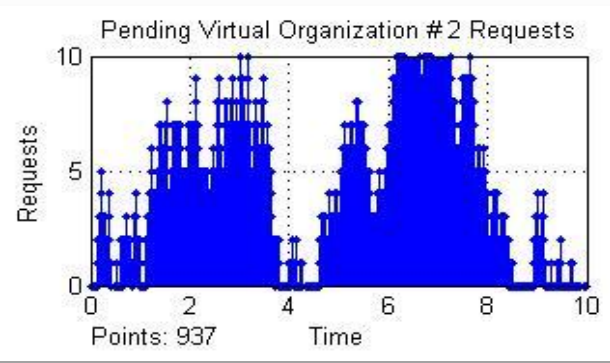

(b)

Figures 9 Description of Jobs queued and availability of resources in the VO2

Two types of capacity queues for both organizations were considered. For the finite one, when queues are saturated, applications that have no place in the queue are not processed and processing is aborted. The same behavior was observed with increasing frequency with respect to time between jobs arrivals.

\section{Phase 2}

In this phase, we simulated a solution using the multi-agent model proposed as the original contribution to this research. The architecture of the simulated grid environment is similar to that presented in phase 1 . The values of the parameters evaluated were retained. The same distributions were used in order to make a comparative analysis under identical conditions. The MATLAB / SIMULINK model of the proposed integrated multi-agent system architecture is shown in Figure 10.

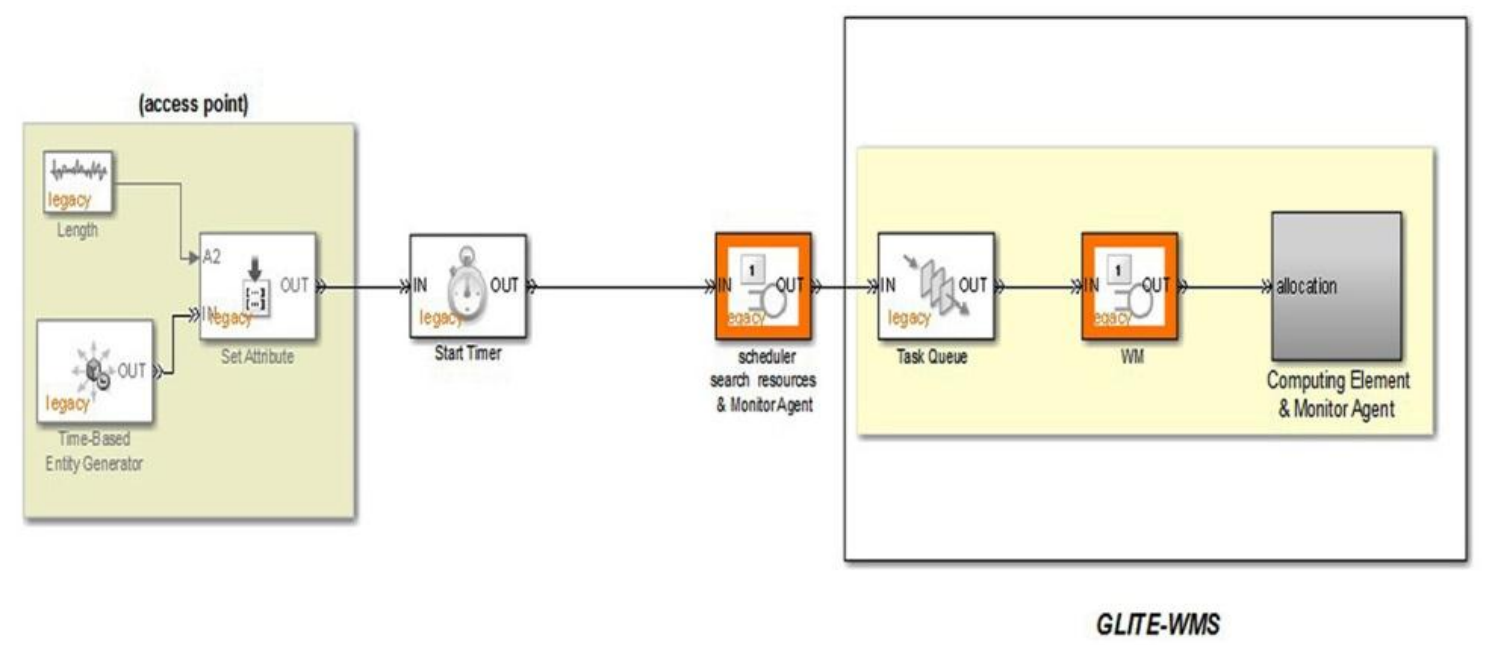

Figure 10 MATLAB/SIMULINK model of multi-agent system architecture, interacting with gLite middleware

Strategy: Load Balancing. Figures $12 a$ and $12 b$ show the VO with a pool 4 computational resources, and Figures $13 a$ and $13 b$ show the VO with pool 8 computational resources. The MATLAB/SIMULINK model to achieve the allocation of resources under the load balancing scheme is shown in the Figure 11.
During this phase the agents are involved in resource management. Agents are logged through the AUI. In order to keep the gLite WMS updated, the local MA starts monitoring the online resources and communicates continuously via an interface with the information Supermarket, to keep the WMS gLite updated. 
Francisco et al.

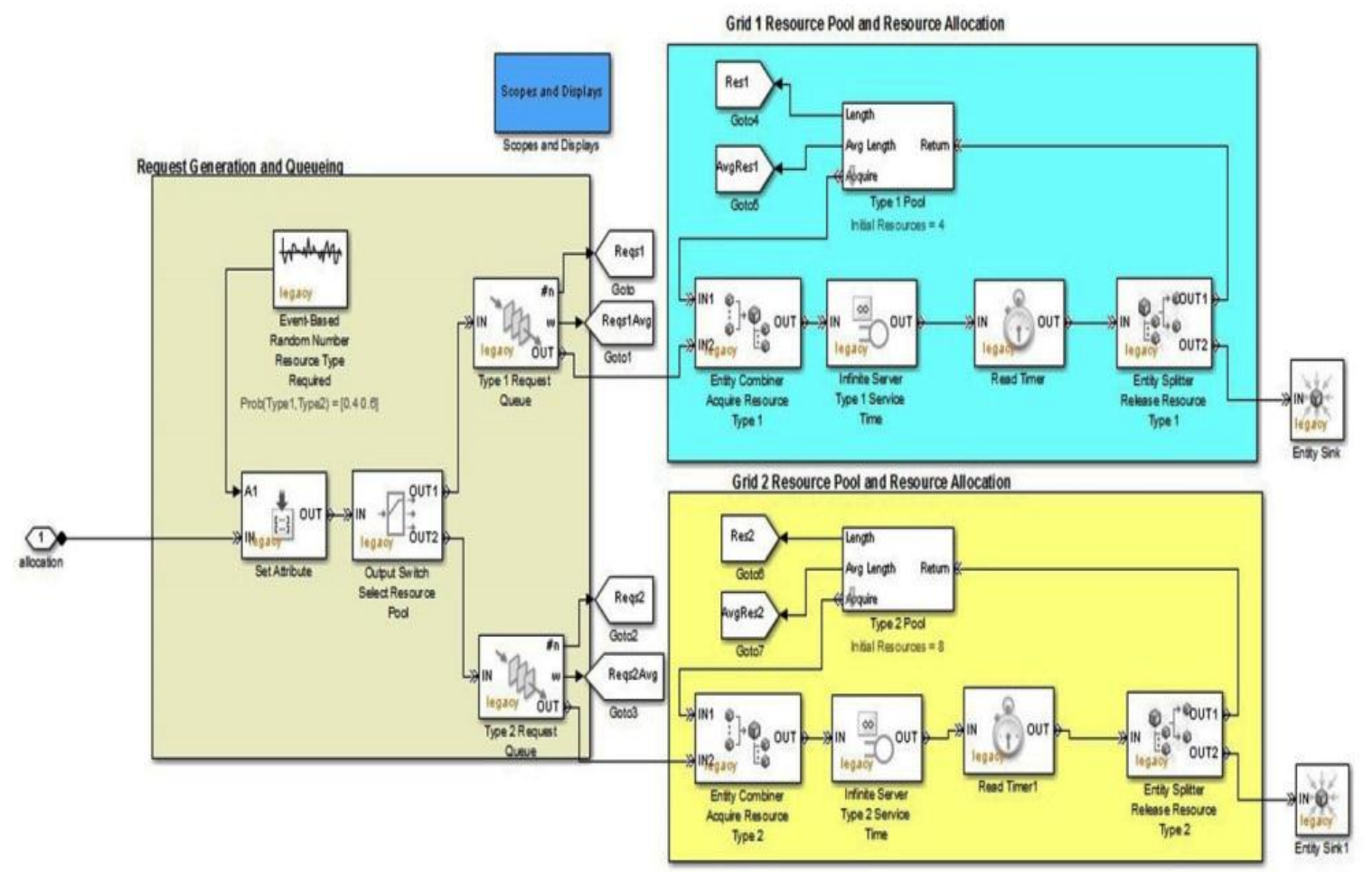

Figure 11 Scheme of the process of resource allocation in the OVs with the integration of the multiagent system (Load Balancing)

The load balancing was implemented by sending $60 \%$ of the applications received to the VO with more resources. The frequency of the time between jobs arrivals increased significantly. The SA, RSA, MA agents began their interactions through the communication interfaces established between them and the WMS. Subsequently, the SA is in charge of transmitting the application requirements to $\mathrm{WM}$ in order to proceed with the allocation of appropriate resources.

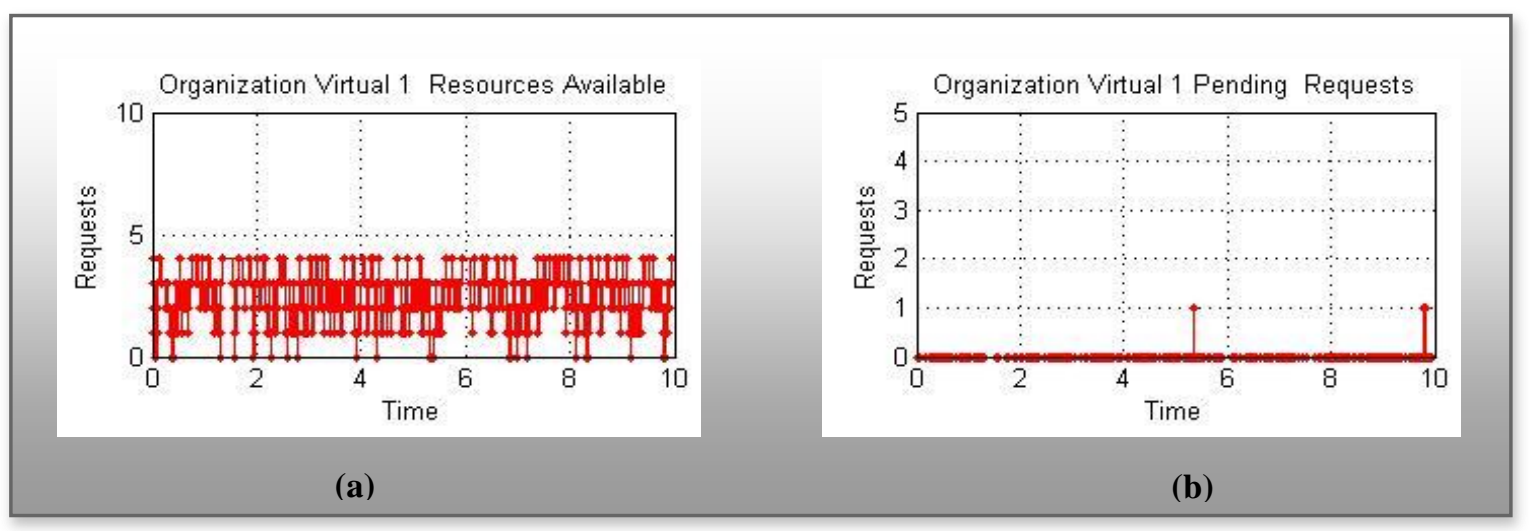

Figures 12 Description of jobs queued and availability of resources in the VO1

From this perspective, we can observe that at all times there are resources available as shown in Figures $12 a$ and $13 a$. Whereas Figure $12 b$ and $13 b$ show no requests waiting in line since they have been dealt with promptly. 


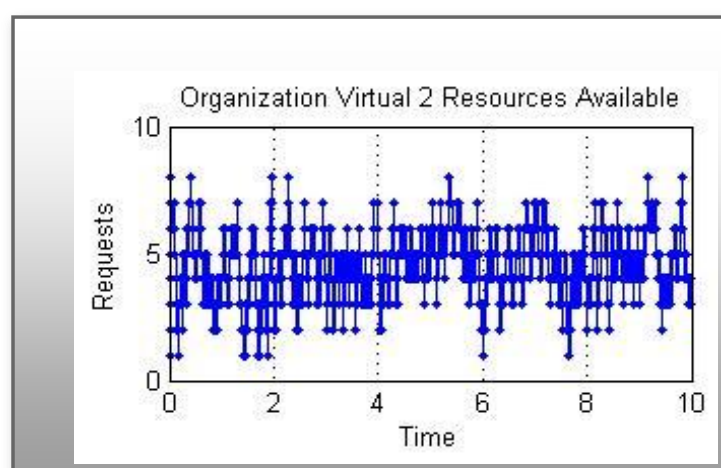

(a)

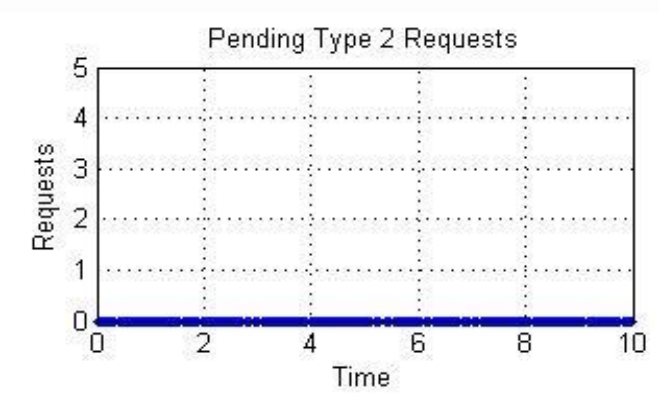

(b)

Figures 13 Description of Jobs queued and availability of resources in the VO2

Under this scheme, resource allocation is performed satisfactorily and the number of entities in line was negligible throughout the simulation period in both domains. Even when varying the queue capacity, finite system performance was maintained.

Strategy: Shortest Route Policy. In the second group of experiments the behavior of the system for the management process of resources was evaluated. We simulated a solution for the SBA in which the SA implements the policy resource selection, i.e. shortest route to effective job submission. Furthermore, the balance between the demand for application resources and the optimal use of all resources was accomplished. For choosing the shortest path, we considered the number of entities in both, the request queue and the number of entities queued in the resource to be assigned. The MATLAB/SIMULINK model is shown in Figure 14. As can be seen in Figures $15 a$ and $16 a$, starting the simulation process shortest path leads to resource entities belonging to the VO1. At that moment the VO2 tends to decrease the number of entities in relation to the tail VO1. Then VO2 becomes the shortest path, and thus the process of allocating resources belonging to that begins.

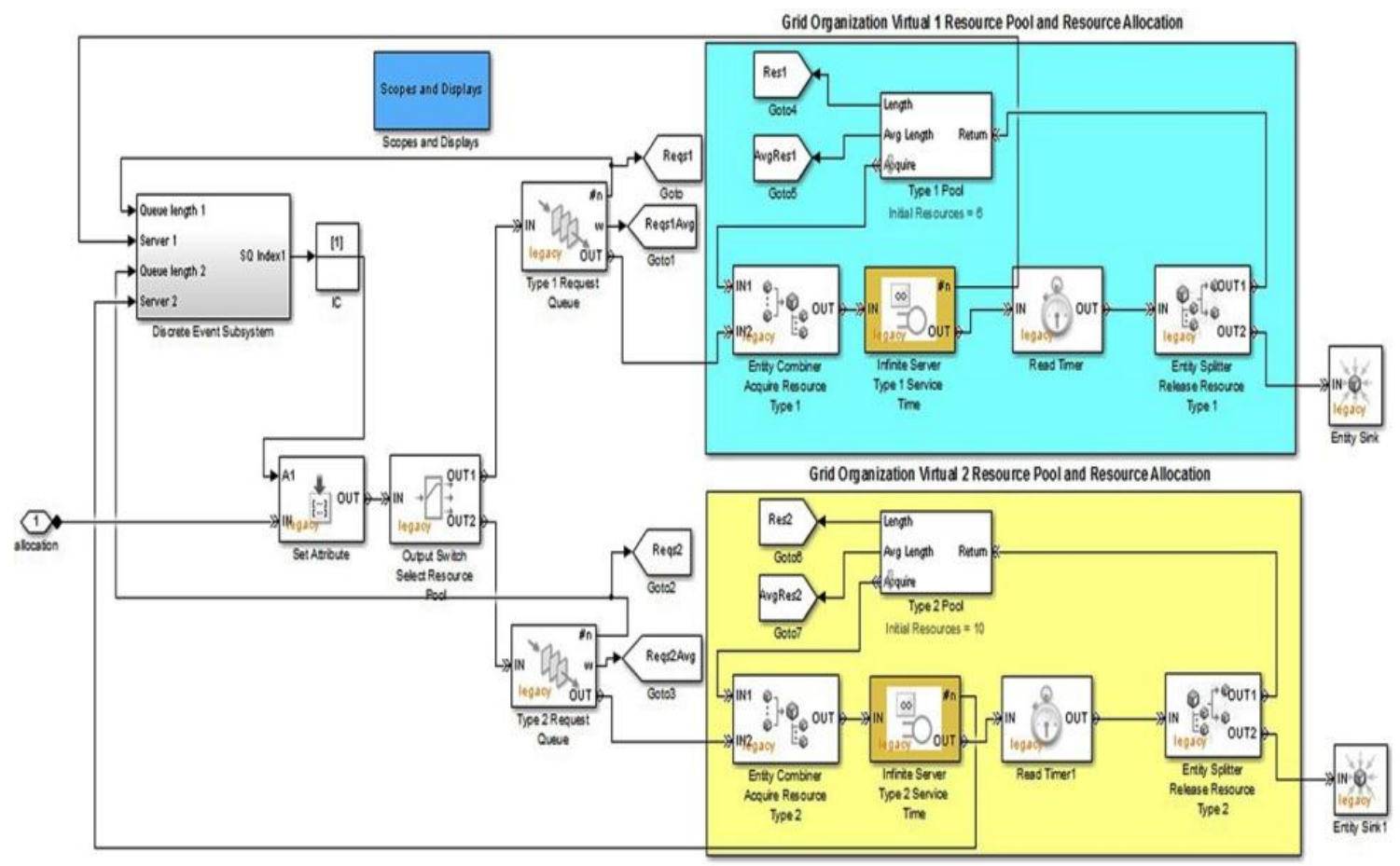

Figure 14 Scheme of the resource allocation process in the OVs shortest route police 


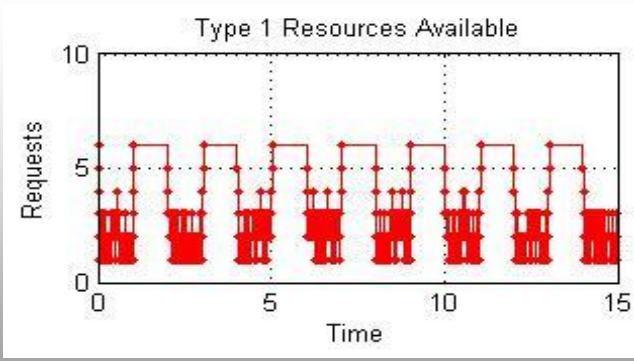

(a)

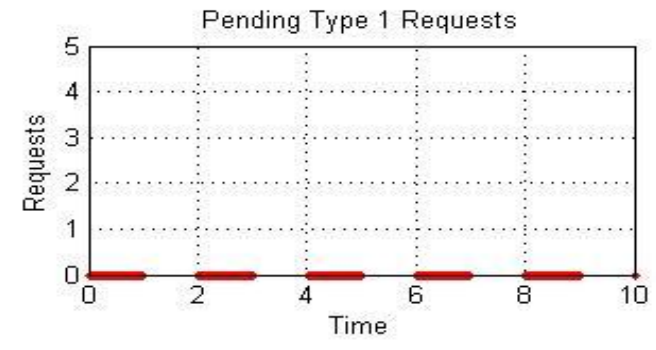

(b)

Figures 15 Description of jobs queued and availability of resources in the VO1

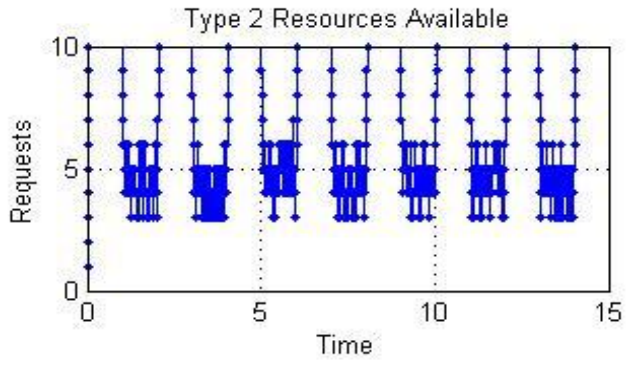

(a)

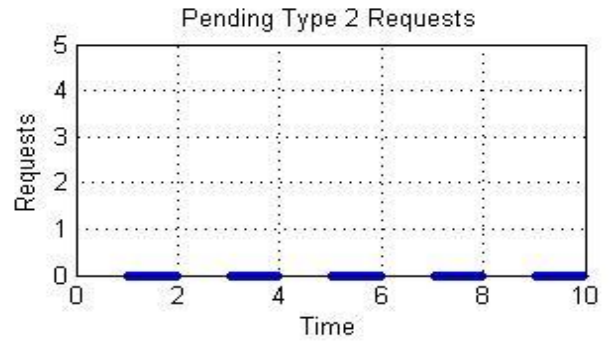

(b)

Figure 16 Description of jobs queued and availability of resources in the VO2

Strategy: Multiple Jobs. Load Balancing. This experiment is considered different types of jobs to be served: Job_Class1 that implements data computationally intensive and require specialized resources to optimize performance; Job_Class3 and Job_Class2 requiring less robust resources. Load balancing is implemented as described above philosophy. The system proceeds to assign the computational resources required in satisfying such requirements VO as shown in Figures 17 and 18.

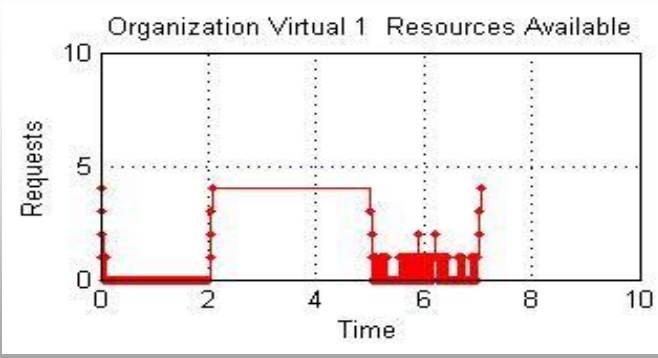

(a)

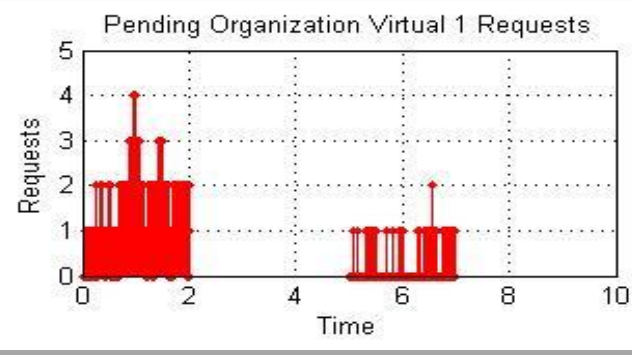

(b)

Figures 17 Description of Jobs queued and availability of resources in the VO1 


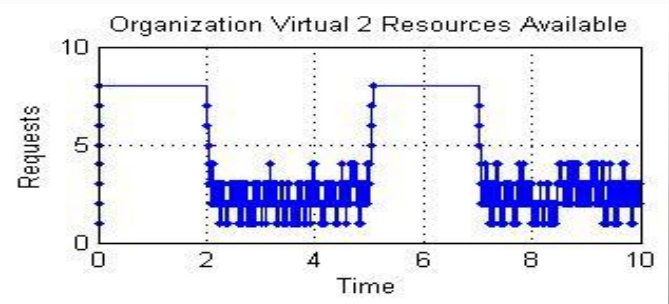

(a)

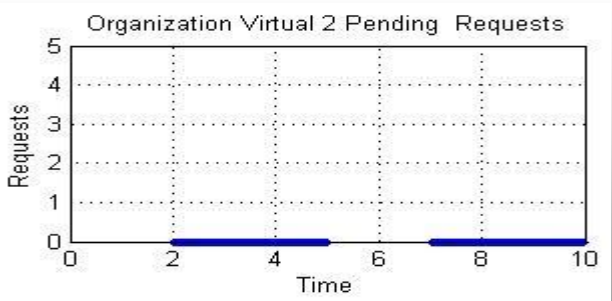

(b)

Figures 18 Description of Jobs queued and availability of resources in the VO2

In both instances, the expected performance of the system is maintained. Service load balancing allows jobs to be efficiently allocated by the local SA thus

avoiding system saturation. The MATLAB/SIMULINK model is shown in Figure 19.

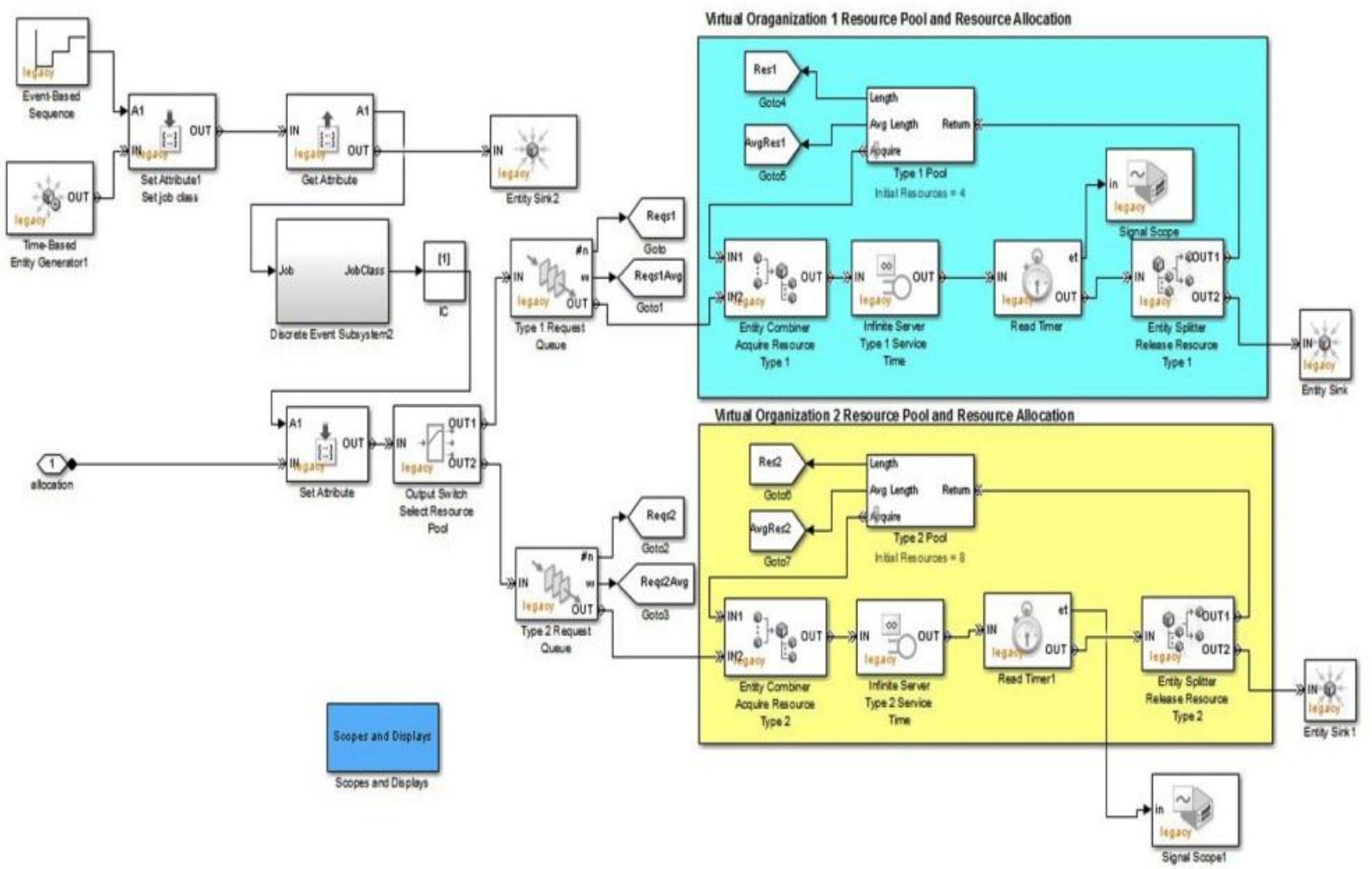

Figure 19 Scheme of the resource allocation process in the OVs (Multiple jobs: load balancing)

Strategy: Control Fault. This scenario was designed to model situations when a server fails; as, for example, when a machine fails and it is subsequently repaired or when a network connection fails and is subsequently restored. The service fault control is implemented when the monitoring service reports a disruption in execution. The model MATLAB/SIMULINK in Figure 20 shows the fault detection process and resource allocation. The results above show the effective resources allocation and balance of the system while maintaining the availability of resources in the VO. All this regardless of the failures in the system. Figure 21 shows the description of Jobs queued and availability of resources in VO1 and VO2 respectively. Figure 22 shows the sequence executions and failures. 
Francisco et al.

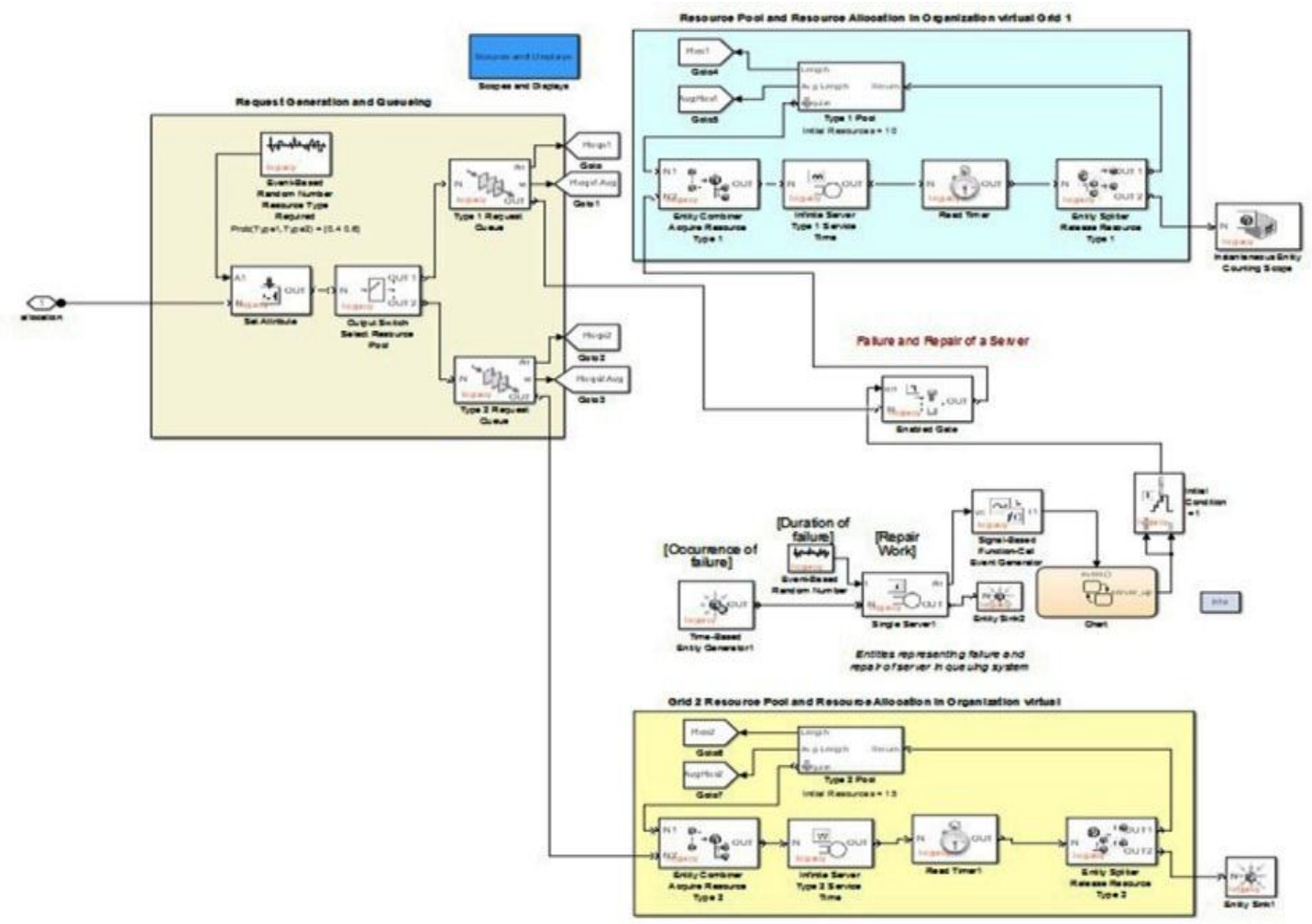

Figure 20 Scheme of the resource allocation process in the OVs (Control fault)
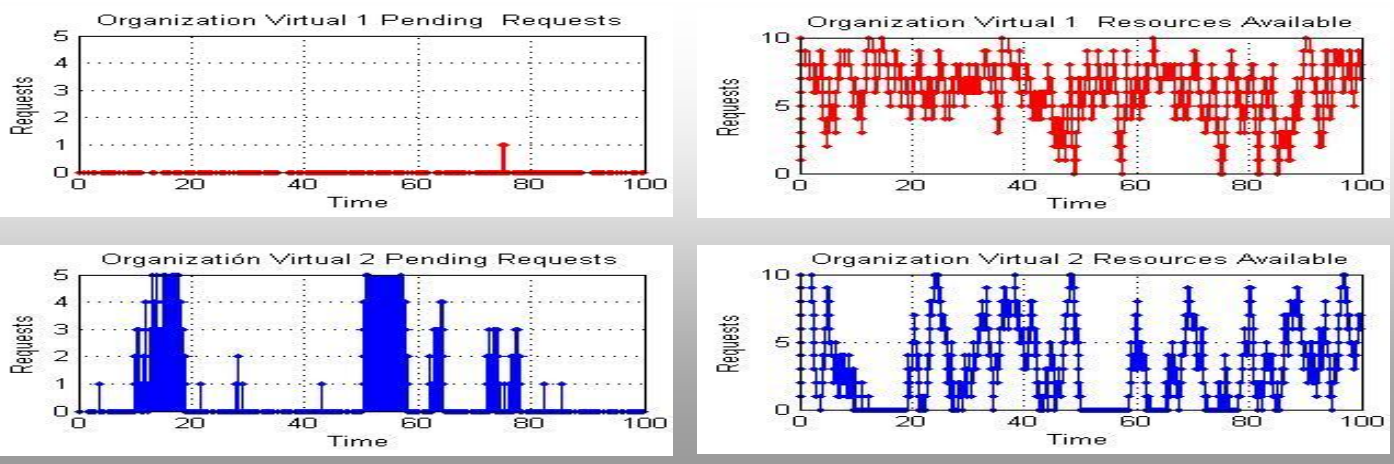

Figure 21 Description of Jobs queued and availability of resources in VO1 and VO2 respectively

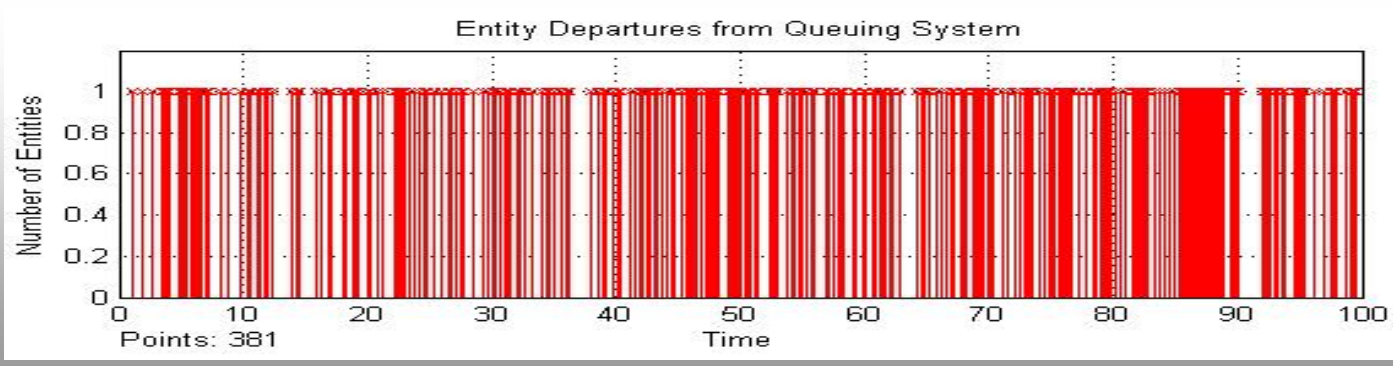

Figure 22 Sequence executions and failures 


\section{Discussion}

The grid gLite middleware architecture presents several limitations for resource management. One type of major failure is when the execution of job aborts because the assigned resource is busy. This failure is due to the non-actual updating of the status of the resource.

The proposed agent-based architecture provides great scope for management and network overload. In the implementation of the simulation model discussed in section 3, some of the advantages of the proposed architecture in relation to the gLite architecture could be evidenced. The values of the parameters evaluated were retained for both simulations and the same distributions were used in order to make a comparative analysis under identical conditions.

\subsection{The gLite limitations}

The gLite information update limitations are a source of inspiration for proposing our agent-based model. The risks of execution abortion, intolerance to failures and a poor distribution of resources are weaknesses that need to be improved and optimized.

We proposed multiagent based architecture model for managing computer resources in a grid. This includes planning, implementation and monitoring for improving the gLite middleware services for tasks involving the running in a Grid. In our model, the use of agents allows the analysis of online information, better distribution of it. Furthermore, an interface with the user is available to through the interface agents. The state of online availability of the computing resources of the different administrative domains that are part of the high performance network is omitted.

In this scheme, it is assumed that the update time of the information about the availability and occupation of the resources is established by the gLite middleware. The resource allocation is done without any prior policy. All resources have the same assignment probability and all requests for resources are answered. If the service time of resources (according to the work they are processed) tends to augment the waiting time and then the congestion in the queues increases. For queue of infinite capacity, congestion occurs. The applications rejected in the queue are not processed so their ejection aborts and the user are informed.

\subsection{Agent-based architecture can be extended at} any of its levels

The agent model is very flexible. New agents can be added and new targets can be attributed to the agents. Agents differ by their position in the hierarchy, but not by their functionality. New and diverse types of equipment can be added to the grid according to the treatment and analysis of the needs of each VO. The hierarchical model solves the problems of scalability. A greater number of agents in the hierarchy will lead to greater activity in the local domain without the intervention of a central agent. In this way, the system scales correctly.

\subsection{The data analysis time can be reduced}

The data analysis time can be reduced. We affirm this since the communication complexity of the system is $<\mathrm{O}(\mathrm{n} 2)$ in the worst case, and by the possibility of adding resources and agents in the grid and a better distribution of activities.

4.4Distribution of the workload and the use of specialized resources of the grid

gLite does not implement any type of distribution of workload. The importance of the use of intelligent agents is that they have the knowledge to distribute and / or balance the processing of the workload through the discovery of available resources and the implementation of policies or heuristics for management.

4.5Knowledge exchange for network management An important advantage of the agent's model is the ability to exchange information between network agents. The use of an agent infrastructure seems to be more attractive when the volume of information analyzed in the network is relatively large.

\subsection{Fault control}

The service is activated when the monitoring agent reports an interruption in the execution. The process of effective allocation of resources in the different available VOs is restarted. The grid middleware does not have this mechanism and in this sense it aborts the execution of the job.

\section{Conclusions}

In this study the behavior of a multi-agent system in a high-speed distributed computer network was evaluated. We showed the advantages of agent-based resource management compared to a gLite grid middleware architecture. 
A detailed study of the impact of incorporating strategies in allocation policies jobs in the overall performance of the grid was presented. When the system is subjected to stress, the results showed that the communication time of each message depended on several factors: number of messages, message size, interconnection infrastructure, and transfer mode. Cases are presented when a centralized database for information about the availability of resources of system latency increase significantly due to the generation of a higher density of messages to the database.

The results suggest that the proposed model (SBA) generates low computational complexity. The results of the simulation presented in the paper showed that the criteria considered, that is, allocation strategies, are robust and stable, and that the system optimizes its ability to different workloads. These results hold even when system conditions were changed and when it was subject to stress. The grid environments provide a promising platform for the efficient execution of complex applications. The proposed architecture based on agents (SBA) provides a highlevel abstraction powerful for resource management. This model may be regarded as a mechanism to minimize network overhead achieving balance in local networks. The SBA is, therefore, a new prototype for resource management in high performance computing environments.

\section{Acknowledgment}

We thank the Center for Parallel and Distributed Computing of Central University of Venezuela (CCPDUCV) for their support in the development of this research.

\section{Conflicts of interest}

The authors have no conflicts of interest to declare.

\section{References}

[1] Czajkowski K. GT resource management and scheduling: the globus perspective. GlobusWorld 2003:13-7.

[2] Pathak M, Bhartee AK, Tandon V. An efficient scheduling policy for load balancing model for computational grid system. Computer Engineering and Intelligent Systems. 2012; 3(7):51-61.

[3] Czajkowski K, Fitzgerald S, Foster I, Kesselman C. Grid information services for distributed resource sharing. In international symposium on high performance distributed computing 2001 (pp. 181-94). IEEE.

[4] Xiao P, Liu D, Qu X. Three-side gaming model for resource co-allocation in grid computing. Journal of Software. 2012; 7(9):2125-32.
[5] Foster I, Kesselman C. The grid 2: blueprint for a new computing infrastructure. Elsevier; 2003.

[6] Bajo J, Corchado JM. Neural systems in distributed computing and artificial intelligence. Neurocomputing. 2017; (231):1-2.

[7] Kiourt C, Kalles D. A distributed multi agents based platform for high performance computing infrastructures. Workshop on parallel and distributed computing for knowledge discovery in databases 2016.

[8] Kiourt C, Kalles D. A platform for large-scale gameplaying multi-agent systems on a high performance computing infrastructure. Multiagent and Grid Systems. 2016; 12(1):35-54.

[9] $\mathrm{Hu}$ J, Gao J. IMCAG: infrastructure for managing and controlling agent grid. In international conference on grid and cooperative computing 2003 (pp. 161-5). Springer, Berlin, Heidelberg.

[10] Yousif A, Abdullah AH, Latiff MS, Bashir MB. A taxonomy of grid resource selection mechanisms. International Journal of Grid and Distributed Computing. 2011; 4(3):107-17.

[11] Yousif A, Nor SM, Abdualla AH, Bashir MB. Job scheduling algorithms on grid computing: state-of-the art. International Journal of Grid and Distributed Computing. 2015; 8(6):125-40.

[12] Utkarsh K, Trivedi A, Srinivasan D, Reindl T. A consensus-based distributed computational intelligence technique for real-time optimal control in smart distribution grids. IEEE Transactions on Emerging Topics in Computational Intelligence. 2017; 1(1):51-60.

[13] Mariotti M, Gervasi O, Vella F, Costantini A, Cuzzocrea A. A DBMS-based system for integrating grids and clouds: anatomy, models, functionalities. In proceedings of the international conference on internet of things and cloud computing 2016. ACM.

[14] Foster I. Globus toolkit version 4: software for service-oriented systems. In IFIP international conference on network and parallel computing 2005 (pp. 2-13). Springer, Berlin, Heidelberg.

[15] Kaur N, Kaur H, Ahuja SP. Analysis of advanced grid resource management models. Research Cell: An International Journal of Engineering Sciences. 2011.

[16] Foster I, Kesselman C. Globus: a metacomputing infrastructure toolkit. The International Journal of Supercomputer Applications and High Performance Computing. 1997; 11(2):115-28.

[17] Open Grid Forum. An Open Global Forum for Advanced Distributed Computing. https://www.ogf.org/ogf/doku.php. Accessed 21 August 2017.

[18] Foster I, Kesselman C, Tuecke S. The anatomy of the grid: enabling scalable virtual organizations. The International Journal of High Performance Computing Applications. 2001; 15(3):200-22.

[19] Luan C, Song G, Zheng Y. An infrastructure for grid job monitoring. In international conference on grid and cooperative computing 2005 (pp. 443-8). Springer, Berlin, Heidelberg. 
[20] Yousif A, Nor SM, Abdullah AH, Bashir MB. A discrete firefly algorithm for scheduling jobs on computational grid. In: Yang XS. (eds) cuckoo search and firefly algorithm. Studies in Computational Intelligence 2014, p. 271-90. Springer International Publishing.

[21] Li J, Zhang GY, Gu GC. A multi-agent based architecture for network attack resistant system. In international conference on grid and cooperative computing 2003 (pp. 980-3). Springer, Berlin, Heidelberg.

[22] Foster I, Jennings NR, Kesselman C. Brain meets brawn: why grid and agents need each other. In proceedings of the third international joint conference on autonomous agents and multiagent systems (pp. 815). IEEE Computer Society.

[23] FIPA ACL Message Structure Specification. http://www.fipa.org/specs/fipa00061/SC00061G.html. Accessed 10 June 2017.

[24] Kim JS, Quang B, Rho S, Kim S, Kim S, Breton V, et al. Towards effective scheduling policies for manytask applications: practice and experience based on HTCaaS. Concurrency and Computation: Practice and Experience. 2017; 29(21):1-15.

[25] Serrano JF, Suros RE. Design of a multiagent system for the management of resources in high speed networks. International symposium on communication of knowledge and conferences 2008 (pp. 71-6). International Institute of Informatics and Systemics.

[26] Foster I, Kesselman C, Nick JM, Tuecke S. Grid services for distributed system integration. Computer. 2002; 35(6):37-46.

[27] Shestak V, Chong EK, Maciejewski AA, Siegel HJ. Probabilistic resource allocation in heterogeneous distributed systems with random failures. Journal of Parallel and Distributed Computing. 2012; 72(10):1186-94.

[28] Jiang Y, Huang J, Ding J, Liu Y. Method of fault detection in cloud computing systems. International Journal of Grid and Distributed Computing. 2014; 7(3):205-12.

[29] Sugavanam P, Siegel HJ, Maciejewski AA, Oltikar M, Mehta A, Pichel R, et al. Robust static allocation of resources for independent tasks under makespan and dollar cost constraints. Journal of Parallel and Distributed Computing. 2007; 67(4):400-16.
[30] Yagoubi B, Meddeber M. Distributed load balancing model for grid computing. African Journal of Research in Computer Science and Applied Mathematics. 2010; 12:43-60.

[31] EGEE Middleware Design Team. EGEE Deliverable 1.4: EGEE Middleware Architecture. https://edms.cern.ch/document/594698/. Accessed 11 July 2017.

[32] http://www.upv.es/sma/teoria/agentes/tesiscif.pdf. Accessed 11 July 2017.

[33] Xiong Z, Zhang X, Liu L. Resource management model and resource discovery algorithm for P2PGrid. Journal of Networks. 2011; 6(8):1187-94.

[34] Wilkinson B, Allen M. Parallel programming: techniques and applications using networked workstations and parallel computers. Pearson Education; 2005.

[35] Foster I, Kesselman C, Nick JM, Tuecke S. The physiology of the grid. Grid computing: making the global infrastructure a reality. 2003, p.217-49.

[36] https://edms.cern.ch/document/674643/1. Accessed 11 July 2017.

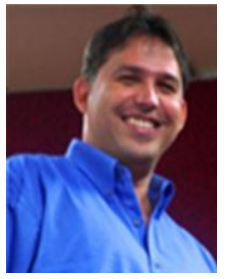

Serrano Juan Francisco is a $\mathrm{Ph} . \mathrm{D}$ research scholar in Computer Science at the Central University of Venezuela. $\mathrm{He}$ is Assistant Professor in the Department of Petroleum Engineering from the University of East Monagas core, Venezuela. He teaches numerical methods for engineers, introduction to programming and simulation oilfields. His research interests include Grid Computing and High Performance Computing.

Email: jfserranom@gmail.com

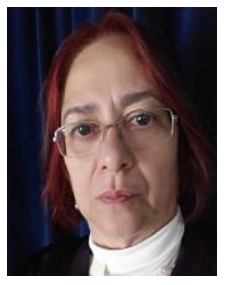

Surós Rina is a $\mathrm{PhD}$ in Computer Science from University of Paris-Sud, Orsay, France. She is a Professor in the department of Computer Science at Central University of Venezuela. His research interests include High Performance Computing, Parallel and Distributed Systems.

Email: rsuros@gmail.com 